This item was submitted to Loughborough's Research Repository by the author.

Items in Figshare are protected by copyright, with all rights reserved, unless otherwise indicated.

\title{
Indoor measurement of photovoltaic device characteristics at varying irradiance, temperature and spectrum for energy rating
}

PLEASE CITE THE PUBLISHED VERSION

http://dx.doi.org/10.1088/0957-0233/21/11/115701

PUBLISHER

(c) IOP Publishing Ltd

\section{VERSION}

AM (Accepted Manuscript)

\section{PUBLISHER STATEMENT}

This work is made available according to the conditions of the Creative Commons Attribution-NonCommercialNoDerivatives 4.0 International (CC BY-NC-ND 4.0) licence. Full details of this licence are available at: https://creativecommons.org/licenses/by-nc-nd/4.0/

\section{LICENCE}

CC BY-NC-ND 4.0

\section{REPOSITORY RECORD}

Bliss, Martin, Thomas R. Betts, and Ralph Gottschalg. 2019. "Indoor Measurement of Photovoltaic Device Characteristics at Varying Irradiance, Temperature and Spectrum for Energy Rating". figshare. https://hdl.handle.net/2134/17796. 


\title{
1 Indoor measurement of photovoltaic (PV) device 2 characteristics at varying irradiance, temperature and 3 spectrum for energy rating
}

\author{
M Bliss, T R Betts and R Gottschalg \\ Centre for Renewable Energy Systems Technology (CREST), Department of \\ Electronic and Electrical Eng., Loughborough University, Loughborough, \\ Leicestershire, LE11 3TU, UK \\ Email: M.Bliss@,lboro.ac.uk
}

\begin{abstract}
The first three dimensional performance matrix for use in photovoltaic (PV) energy rating is reported utilising a novel energy rating solar simulator based on LEDs. Device characteristics are measured indoors under varying irradiance $(G)$, temperature $(\mathrm{T})$ and spectrum $(\mathrm{E})$. This opens the possibility for a more accurate measurement system for energy yield prediction of PV devices especially for devices with high spectral dependency as such as wide bandgap solar cells as it is taking into account spectral changes in the light. The main aspects of the LED-based solar simulator used are briefly described. A measurement method is developed and detailed in the paper, which takes into account the current imperfections in the achievable spectrum. Measurement results for a crystalline silicon solar cell are used to demonstrate the measurement approach. An uncertainty analysis of the measurement system is given, resulting in an overall absolute uncertainty of $4.3 \%$ (coverage factor $\mathrm{k}=2$ ) in maximum power measurements at $765 \mathrm{~W} / \mathrm{m}^{2}$ irradiance with scope for further improvements.
\end{abstract}

Keywords: Indoor measurement, performance matrix, energy rating, LED-based solar simulator, photovoltaics, solar cell

\section{Introduction}

26 Photovoltaic (PV) devices are typically characterised in a solar simulator that measures the power at 27 standard test conditions (STC), i.e. with air mass (AM) $1.5 \mathrm{G}$ spectrum, $1000 \mathrm{~W} / \mathrm{m}^{2}$ light irradiance and $2825^{\circ} \mathrm{C}$ test device temperature [1]. This allows good comparability between devices of the same 


\section{Indoor Measurement of GTE-matrix for Energy Rating}

1 manufacturer and technology, but realistic operating performance may vary, especially between 2 devices of different technology. The customer, however, requires energy from a photovoltaic 3 installation while most of the characterisation and pricing is done at STC - which is a misalignment of interests in the market place. The product user and investor are much more interested in the actual financial return and energy production of their photovoltaic technology of choice, typically dependent on energy yield. This mismatch between the performance indicators of manufacturer and user results in energy yield prediction of PV devices gaining in importance. Energy yield prediction, unlike power rating, takes realistic variations of environmental conditions into account. Its outcome is the number of $\mathrm{kWh}$ generated at certain sites. This requires better information on how a device performs in different climatic conditions. Energy yield prediction is a complex process that requires measured PV device characteristics at varying conditions as well as meteorological data from the site to be investigated. Both, meteorological data and device characteristics are fed into a procedure that predicts the energy yield. This paper focuses on the measurement of the device characteristics required for an energy prediction, the specific conditions are site dependent and thus only generic characteristics are measured here.

Characteristics of PV devices for energy yield prediction are either measured outdoors or indoors in solar simulators. Typically, a matrix of different irradiances $(\mathrm{G})$ and temperatures $(\mathrm{T})$ is generated. Energy yield predictions derived from outdoor measurements can be very accurate [2], but may require a long time. Especially, if considering all spectral variations seen at a site this can take months. Even then, separating the effects of irradiance, temperature, spectrum and angle of incident, which is required to enable a site-to-site translation, is very difficult. Indoor measurements need correction of the G-T measurements for the effects of spectrum (E). This can lead to uncertainties in the energy prediction. Especially multi-junction and wide bandgap solar cells are non-linear with spectrum. Thus the accuracy of yield predictions depends on how much a device is affected by these spectral variations. Spectral effects on energy yield prediction do not average out as in many locations an on average bluer spectrum is observed ([3] and [4]). Some materials, as such as amorphous silicon devices ([5], [6] and [7]) show a further effect on the fill factor and one should expect additional uncertainties in the yield prediction. The spectral mismatch correction [8] is thus only of limited applicability. Thus it appears advisable to measure the effects of varying spectrum directly.

A realistic set of measurements would contain a matrix of measurements taken at all realistic conditions for $\mathrm{G}, \mathrm{T}$ and $\mathrm{E}$, as they would be seen at the given site to be investigated. This has not been possible to date, because solar simulators did not provide the variability of spectral conditions required. An LED-based solar simulator has been developed that can closely reproduce operation conditions seen outdoors, i.e. varying spectrum, irradiance and device temperature. The variation in clear sky air-mass (AM) is demonstrated here. There is no problem to include any measured dataset 


\section{Indoor Measurement of GTE-matrix for Energy Rating}

1 here, albeit there is a dearth of long-term measured spectral data for sites. The dataset measured is the

2 device characteristic required for including non-linear spectral effects in an energy yield prediction.

3 Measurements taken in a simulator take a shorter time than outdoors-based characterisations, as

4 measurement conditions can be controlled and one does not have to wait for the weather conditions

5 and seasonal variations.

6 Multi-source solar simulators with abilities of changing the spectral output as such as presented in [9]

7 and [10] are used to-date primarily to calibrate the performance of multi-junction solar cells. They

8 change the balance of chunks of the spectrum, i.e. the bands $300-800 \mathrm{~nm}$ in one go, but they are not

9 able to reproduce the intricacies of variable air mass. They may be used for spectrometric

10 characterisation as well as optimisation of device structures [11]. The LED-based solar simulator

11 prototype improves on this as the system provides a wider variability of spectral conditions with a

12 better control of the shape of the sunlight spectrum with is variations.

13 One factor not considered in this paper is the angle of incidence. One could argue that this would be a 14 further dimension in the measurements but on the other hand one could include this in the incident 15 spectral irradiance. Thus, this measurement could be taken in an independent measurement system.

16 Previous work on the LED-based simulator prototype was very much concentrated on the system 17 development, analysis and optimisation [12] and [13] at STC conditions. This paper presents the 18 extension to energy rating, i.e. variable temperature, irradiance and spectrum. In the following, details 19 of the LED-based solar simulator are given. The measurement method used is described in detail. The 20 effects of the spectrum on a c-Si solar cell are reported and contrasted to reported outdoor conditions. 21 To evaluate how robust the measurements taken by system are, uncertainties in the measurements are 22 analysed and discussed.

\section{Measurement system}

24 All measurements presented here have been carried out using the LED-based solar simulator prototype developed at CREST, previously reported in [12] and [13] and modified for this work. The system utilises 8 different LED types (colours) to cover the light spectrum from ultraviolet at $375 \mathrm{~nm}$ to red at $680 \mathrm{~nm}$. Halogen light sources are used to cover the infrared part of the output spectrum. All colours are controllable separately. The total output spectrum can be adjusted by independently changing the light intensity of each colour. Thus, a flexible spectral and light intensity control is achieved. Additionally, LEDs allow intensity adjustments with only minimal changes in spectral output, which is of advantage for accurate intensity changes at the same spectrum. The simulator is rated after IEC 60904-9 [14] as a class BAA (a spectral match class B, irradiance non-uniformity A and temporal stability of class A) when measuring solar cells within an area of up to $60 \times 60 \mathrm{~mm}^{2}$. 
1 To improve current-voltage (I-V) tracing and measurement accuracy, a new in-house developed 42 quadrant power supply with an I-V tracing range of $\pm 10 \mathrm{~V}$ and $\pm 1.8 \mathrm{~A}$ has been implemented into the 3 system. A further modification of the solar simulator was an upgrade of the water cooling PV device

4 temperature control system to a peltier based cooler/heater. This is capable of regulating the device 5 temperature faster and more accurately from $0^{\circ} \mathrm{C}$ to $80^{\circ} \mathrm{C}$ in $0.1^{\circ} \mathrm{C}$ steps. Albeit, in praxis only 6 temperatures down to $15^{\circ} \mathrm{C}$ are used due to condensation on the device's surface. The remaining air 7 gaps between the device under test and the peltier cooling system are filled with thermal gap filling 8 sheets. This reduces the thermal resistance from the peltier stage to the device and ensures that the 9 temperature distribution on the device is even.

\section{Measurement method}

11 The measurement method used in this work consists of three main steps (Figure 1). The first is to 12 define the measurement ranges and points in the G-T-E matrix. The last two steps are repeated for all 13 selected reference spectra and include the adjustment of the simulator output spectrum and the measurement the GT-matrix.

\section{Define measurement ranges}

GTE measurement matrix

Choose first/next sunlight spectra

Reference sunlight spectrum

Calculating and adjusting simulator spectral output

Simulator spectrum control

Irradiance-Temperature (G-T) matrix measurement

G-T matrix at chosen spectra

Measured under all spectra?

TRUE

FALSE

Test device G-T-E device

performance matrix

Figure 1: Basic measurement method for measuring a G-T-E device performance matrix

\subsection{Defining measurement ranges}

When defining the measurement ranges, G-T-E matrix measurement points should be relevant to realistic conditions, i.e. points pertinent to what is seen outdoors should be chosen. This is important 


\section{Indoor Measurement of GTE-matrix for Energy Rating}

1 because the points chosen can have a large impact on the final accuracy of the energy yield 2 calculation. Also the number of measurements can be very large. It is dependent on how many 3 different spectra, intensities and temperatures are chosen. The spectra chosen can be either simulated 4 (e.g. SMARTS [15]) or measured. They would have to be classified by additional factors such as air mass, clearness index and cloud cover. The choice of conditions for good accuracy in energy rating is not covered within this work, as there is a lack of measured data. It should be defined in the proposed energy rating standard IEC 61853 [16].

\section{$8 \quad 3.2 \quad$ Calculating and adjusting simulator spectral output}

9 Prior to the measurement of a light intensity and temperature (G-T) matrix, the solar simulator output 10 spectrum must be adjusted to the chosen reference spectrum. This is done by first calculating the 11 required irradiances of each available light source type and then setting the acquired values in the solar simulator.

13 The intensity configuration of each light source colour either can be acquired manually or else with help of a fitting algorithm that minimises the deviation between the required sunlight spectrum and the spectrum in the solar simulator. Input parameters for the fitting algorithm are the spectral outputs of the light sources in the solar simulator. The parameter to minimise would be the standard deviation over wavelength between reference and combined simulator spectra. Adjustable parameters are the intensity factors of the light sources. In the presented case, the intensity of the halogen light sources was fixed at $100 \%$ during the adjustments of the output spectrum to reduce uncertainties from spectral changes at this point, as the system has been calibrated at this intensity and thus the halogen spectrum is best represented. The spectral variations of halogen lights when controlling intensity are clearly non-ideal and make the case for an all-LED solar simulator.

If the test device is a multi-junction device, it is important to ensure that the junction current balance is the same as it would be under the reference spectrum. This ensures that the correct fill factor, and thus maximum power, are measured. It also means that the simulator's light source intensities additionally need to be slightly re-adjusted for the correct balance. This can be done with help of different methods of which some of them can be found in [17], [18] and [19].

Regarding single-junction devices, once the intensity factors of the light sources are known, the simulator output spectrum can be adjusted with help of the reference cell's spectral response (SR) curve. The device under test can also be used for adjusting the output spectrum, which has been done during this work as is eliminates uncertainties due to spectral mismatch between the test and reference device. The intensity of each of the light sources in the solar simulator is adjusted separately until the theoretical and real measured short circuit current on the test cell match. The theoretical value is calculated using the relative spectral output of the light source, its intensity factor and the SR of the 


\section{Indoor Measurement of GTE-matrix for Energy Rating}

1 test device. At the end of the light adjustment, the same short circuit current as generated for the reference spectrum should be measured on the test device.

3 It is also possible to use a spectroradiometer to check the spectrum and set the light source intensities.

4 In the presented case this was not done and achieved spectra were assumed based on previous spectroradiometer measurements. The difference between the actual spectrum and the estimated spectrum should not be significant as repeatability tests have shown. Thus the main uncertainties are due to SR measurements of the test device and the relative spectral output calibrations of the light sources at full intensity.

\section{$3.3 \quad$ G-T matrix measurement}

10 Once the solar simulator light spectrum has been adjusted, a G-T matrix at this spectrum can be measured. High measurement accuracy is achieved by ensuring spectral stability of the light sources used in the simulator (i.e. minimal spectral variations of light sources when changing intensity). The intensity in the simulator can then be changed by adjusting all the intensity of all light sources to the same degree with regard to their nominal intensity, leaving the actual output spectrum unchanged. If light sources change spectrum to a large degree, a re-adjustment of the solar simulator spectrum or a mismatch correction is required for each intensity step.

The test device's I-V curves are measured and the reference cell's short circuit current $\left(\mathrm{I}_{\mathrm{SC}}\right)$ can be used to determine the actual light intensity during the measurement. During the measurements presented here, the test device has been used as a self reference. In prior the device's short circuit current and SR have been calibrated. This was done to correct for the effects of spectral shift of the halogen light sources. This one shifts into the infrared region when reducing the intensity because of lower operation temperature of the filament. Nevertheless, self referencing introduces additional uncertainties, as it relies on the linearity of the test device and its spectral response measurement. Self reference was used only at a device temperature of $25^{\circ} \mathrm{C}$ to not eliminate the effects of device temperature changes in the G-T matrix measurement. For all other temperatures in the G-T matrix the same irradiance was assumed, which did not contribute significantly to uncertainty, as the simulator has a good repeatability of light conditions as stated in [13].

Furthermore, in order not to eliminate the influences of spectrum in the G-T-E matrix, self referencing was carried out with respect to the reference sunlight spectrum used in the G-T matrix measurement. The conversion from measured $\mathrm{I}_{\mathrm{SC}}$ to measurement irradiance was recalculated for each spectrum used in this study. This was done by first calculating the theoretical short circuit current $\mathrm{I}_{\mathrm{SC}, \mathrm{R}}$ of the test device under reference sunlight spectrum $E_{R}$ with equation 1. The final measurement irradiance $\mathrm{G}_{\mathrm{M}}$ is then calculated as given in equation 2 . 


$$
\begin{gathered}
I_{S C, R}=A \int S_{T}(\lambda) E_{R}(\lambda) d \lambda \\
G_{M}=G_{R} \frac{I_{S C, M}}{I_{S C, R}}
\end{gathered}
$$

1 A is the test device area, $\mathrm{S}_{\mathrm{T}}$ is the spectral response of the device under test, $\mathrm{I}_{\mathrm{SC}, \mathrm{M}}$ is the measured

2 short circuit current and $G_{R}$ is the irradiance of the reference spectrum $E_{R}$.

3 To accurately change the intensity in the solar simulator, without changing spectrum, the LED-based

4 solar simulator utilises a calibration curve for each light source of the light source intensity versus 5 drive current ( $G$ vs. $\left.I_{D}\right)$. This is used to calculate the correct drive current required to achieve a new 6 setting at which the relative intensities of the light sources are changed equally. The calibration was 7 carried out with a c-Si reference cell at background illumination from other LEDs to reduce possible 8 uncertainties arising from increasing nonlinearities at low light levels. This reduces control errors 9 arising from the slightly non-linear G vs. $I_{D}$ behaviour of the LEDs. With regards to the halogen light 10 sources this calibration ensured that at least the current generated by the light is as much as possible 11 linearly changing with the control even so the spectrum is shifting (e.g. for a setting of 5\% halogen 12 light intensity, the drive current of the halogens is controlled at $55 \%$ ). Calibrations were done at the 13 same warm-up times used for the I-V measurements.

\section{Measurement configuration}

15 The measurement ranges in the G-T-E matrix (see Figure 2) have been defined as following:

- 13 intensities ranging from $5 \%$ to $100 \%$ of the highest irradiance possible 


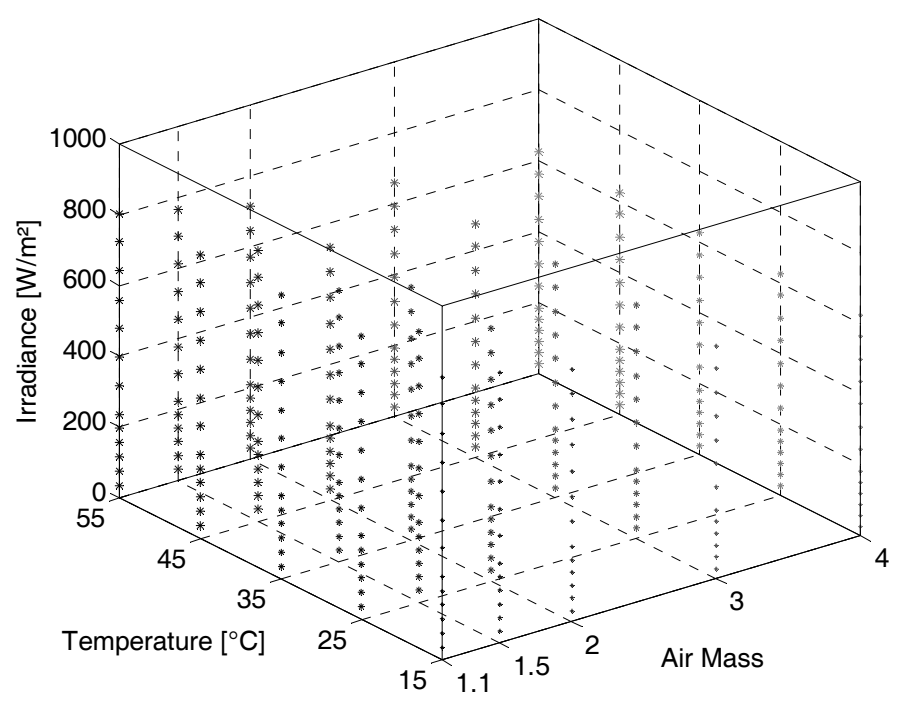

Figure 2: 3D graph of the measurement points in the GTE-matrix; 325 points made up of 5 spectra, each with 13 measurement intensities and 5 device temperatures

Figure 2 shows the measurements points at which the device I-V characteristic has been measured. The measurement points have been chosen for demonstration purposes only. Some of the conditions are not immediately intuitive to occur, but considering rapidly changing cloud cover would make them possible. This includes extreme points as such as AM 1.1 at $800 \mathrm{~W} / \mathrm{m}^{2}$ and $15^{\circ} \mathrm{C}$ or $\mathrm{AM} 4$ at $50 \mathrm{~W} / \mathrm{m}^{2}$ and $55^{\circ} \mathrm{C}$. More likely is a condition as such as $\mathrm{AM} 1.1$ at $800 \mathrm{~W} / \mathrm{m}^{2}$ and $55^{\circ} \mathrm{C}$. Nevertheless, including extreme points into the G-T-E performance matrix can only aide the final energy yield calculation and the time required for different measurements is reasonably short so that it did not appear to be detrimental to measure these points as well. It would also have been better to measure conditions as such AM 1.1 at $1100 \mathrm{~W} / \mathrm{m}^{2}$, but the prototype simulator is not capable of reproducing the 4 sunlight spectra below AM 4 at full intensity. Instead the simulator light was adjusted to the 5 maximum possible intensity. Table 1 shows a comparison between the normal sunlight irradiance and 6 the effective maximum irradiance on the test device reached in the simulator.

Table 1: Irradiance comparison between the reference sunlight spectra used and the effective maximum achieved irradiance in the solar simulator with regard to the measured c-Si solar cell; the last two columns compare the irradiance of sunlight and simulator light in the

\begin{tabular}{ccccc}
\multicolumn{5}{c}{ range of 300-1100nm } \\
\hline \multirow{2}{*}{$\begin{array}{c}\text { Solar } \\
\text { spectrum }\end{array}$} & $\begin{array}{c}\text { Sunlight } \\
(280-4000 \mathrm{~nm})\end{array}$ & $\begin{array}{c}\text { Simulatiance }\left[\mathrm{W} / \mathrm{m}^{2}\right] \\
\text { Effective }\end{array}$ & $\begin{array}{c}\text { Sunlight } \\
(300-1100 \mathrm{~nm})\end{array}$ & $\begin{array}{c}\text { Simulator } \\
(300-1100 \mathrm{~nm})\end{array}$ \\
\hline AM1.1 & 1047 & 801 & 844 & 678 \\
AM1.5 & 997 & 765 & 802 & 648 \\
AM2.0 & 903 & 714 & 721 & 600 \\
AM3.0 & 721 & 659 & 569 & 549 \\
AM4.0 & 599 & 625 & 466 & 517 \\
\hline
\end{tabular}




\section{Indoor Measurement of GTE-matrix for Energy Rating}

1 The reference spectra that have been chosen for this work are all except for AM1.5 simulated with

2 SMARTS for a device that is mounted in a fixed plane with 45 degree tilt and 7 degrees east-of-south.

3 All other input parameters have been set as given in the IEC60904-3 [20]. In case of AM1.5 the standard spectrum has been used. The simulator output spectrum was set to match the reference spectrum as closely as possible (see Figure 3 for AM1.1, AM2 and AM4). The largest deviations between reference and output spectra were found in the $700 \mathrm{~nm}$ to $800 \mathrm{~nm}$ region with up to $37 \%$ lower intensity (AM1.1 being the worst case) using the same wavelength binning as defined by the IEC 60904-9 [14], which leads a class B spectral match. The large deviation is due to low adjustability in the area from red to infrared, as only the halogen lights produce light in this region. As mentioned in the previous section 3.1, the spectra are here assumed to be as simulated in the fitting algorithm.

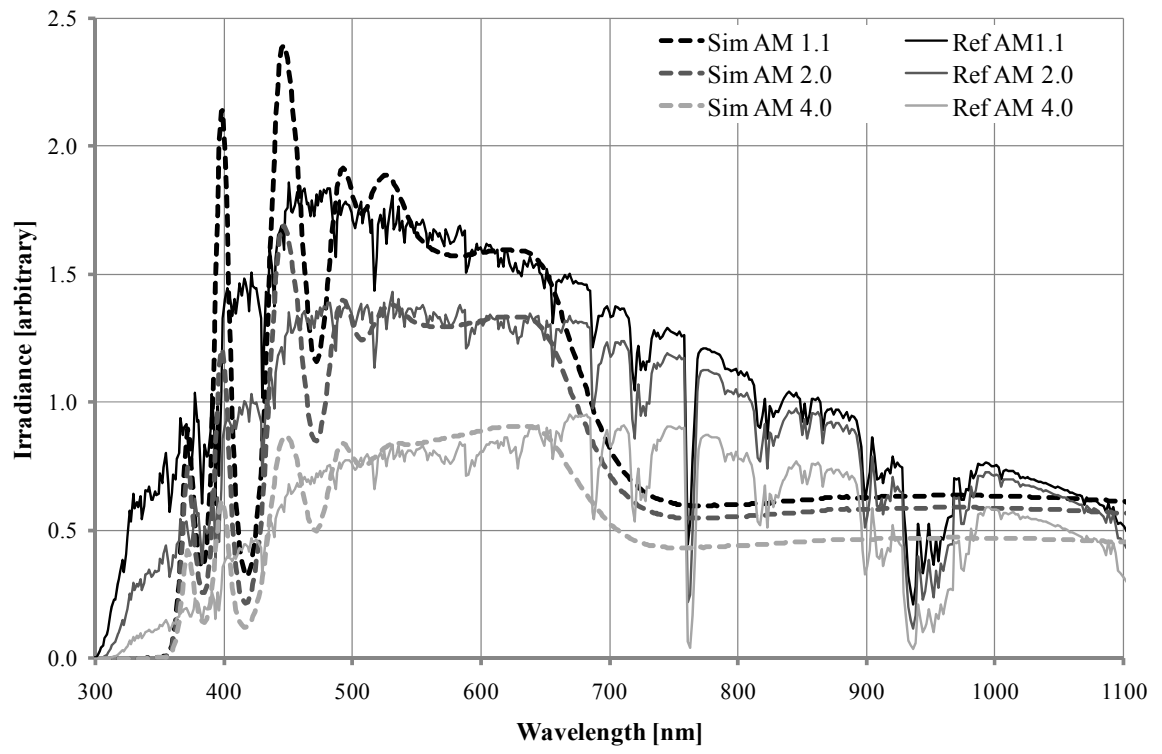

Figure 3: Reference and simulator spectrum; all output spectra used are within class B with the largest deviations in the $700 \mathrm{~nm}$ to $800 \mathrm{~nm}$ region; simulator output spectra are scaled to the reference sunlight spectra to better illustrate the spectral match

The G-T-E matrix measurements have been carried out on a non-encapsulated $30 \times 30 \mathrm{~mm}^{2}$ single junction mono-crystalline silicon solar cell fabricated at CREST. Each I-V curve was measured from short circuit condition to open circuit voltage $\left(\mathrm{V}_{\mathrm{OC}}\right)$ with a resolution of 200 points. The halogen light sources were given a slow warm-up time varying from $3 \mathrm{~s}$ at $100 \%$ intensity to $15 \mathrm{~s}$ at $5 \%$ of their full intensity. This was done to allow them to reach their maximum stability, which takes considerably longer at lower drive currents, as the filaments needs to reach its temperature for intensity as well as stability purposes. After the warm-up of the halogen light sources all LEDs were driven in a long rectangular pulse over $45 \mathrm{~ms}$, allowing a $25 \mathrm{~ms}$ warm-up and a period of $20 \mathrm{~ms}$ for the actual I-V measurement $(0.1 \mathrm{~ms}$ per point). I-V measurements were taken every 90 seconds. This allowed the light sources and electronics cool down and the solar cell temperature to re-stabilize and thus thermal 


\section{Indoor Measurement of GTE-matrix for Energy Rating}

1 influences were minimised. The solar cell operation temperature was changed over a time of about 5

2 minutes, allowing for temperature regulation and settling. A complete G-T matrix took about 2 hours

3 measurement time.

4 The external quantum efficiency of the test device was measured in a filter based spectral response measurement system developed at CREST. Details about the system have been reported in [21]. The lamp and optical set-up have since been optimised and a LED bias light has been fitted. The device was measured at approximately 0.1 suns and compared against the SR of a reference cell (CREST's reference devices are traceable to the JRC-ESTI ${ }^{\mathrm{i}}$ ). An absolute calibration of the SR was done in a Pasan solar simulator against the calibrated $\mathrm{I}_{\mathrm{SC}}$ of the same reference cell with mismatch correction applied.

11 It was stated earlier, that self-reference was used for measuring irradiance during the test sequence. To ensure that did not introduce significant measurement errors, the short circuit current linearity was determined utilising the two lamp method given in IEC 60904-10 [22] and further explained in [23]. The linearity of the device was better than $\pm 1.7 \%$ at intensities below $100 \mathrm{~W} / \mathrm{m}^{2}$ and within $\pm 0.9 \%$ above this, which meant that there was no problem to use the test device as self reference.

\section{Measurement results}

\section{$17 \quad 5.1 \quad$ Spectral behaviour}

18 The unique feature of this system is the variable spectrum, which allows for the first time to investigate spectral effects in a controlled environment. This effect has to date been largely investigated by outdoor measurements (such as in [24] and [25]), where unfortunately all effects are correlated. There are some measurement series on multi-junction devices being reported (such as in [9], [10] and [11]), but none of these were able to approximate the spectral shape yet, only the balance

23 between junctions.

Even in the case of crystalline silicon devices, which are typically seen as being unaffected, a change in efficiency can be observed. As illustrated in Figure 4, increasing air mass results in a small increase in short circuit current over irradiance $\left(\mathrm{I}_{\mathrm{SC}} / \mathrm{G}\right)$. Similar behaviour was also observed in outdoor measurements as shown in [24] and [25]. This is due to the sunlight spectra (and simulator light spectra) changing at a higher rate in the ultraviolet to red $(300-650 \mathrm{~nm})$ region than in the near infrared region, where the spectral response of this c-Si solar cell has its peak. The exact relationship of the possible gain of Isc/G with red-shifting of the spectrum obviously depends on the SR of the device under test. The effect in change of $\mathrm{I}_{\mathrm{SC}} / \mathrm{G}$ affects the device efficiency. Thus even crystalline devices can gain efficiency in red rich, high air mass spectra (Figure 5). 
Indoor Measurement of GTE-matrix for Energy Rating

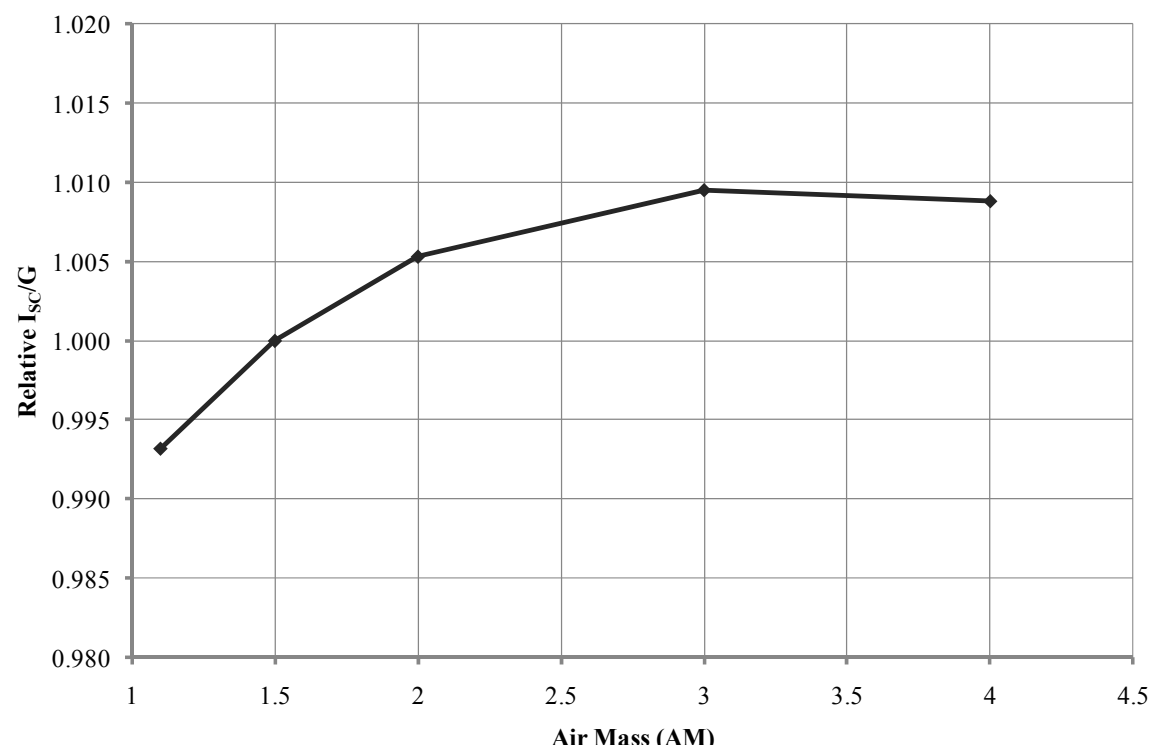

Figure 4: Relative $\mathrm{I}_{\mathrm{SC}} / \mathrm{G}$ versus air mass (AM) normalized to the measurement at AM1.5 spectrum

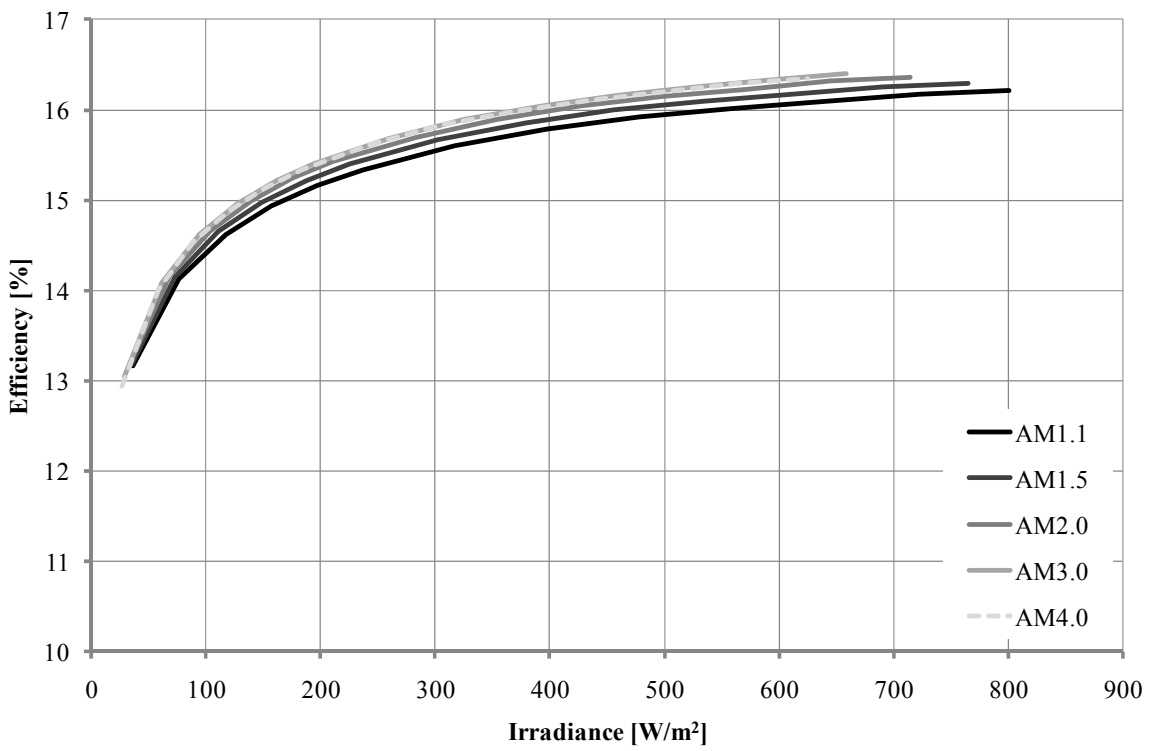

Figure 5: Efficiency versus $\mathrm{G}$ with increasing air mass at $25^{\circ} \mathrm{C}$ device temperature; efficiency increases with air mass

7 As visible in Figure 6 and 7, the influencing factor on the increase in efficiency at higher air mass is 8 the current at maximum power point (MPP). MPP voltage is not visibly affected by spectral changes.

9 It is dominated by light intensity changes. There is no discernable effect on the FF. 


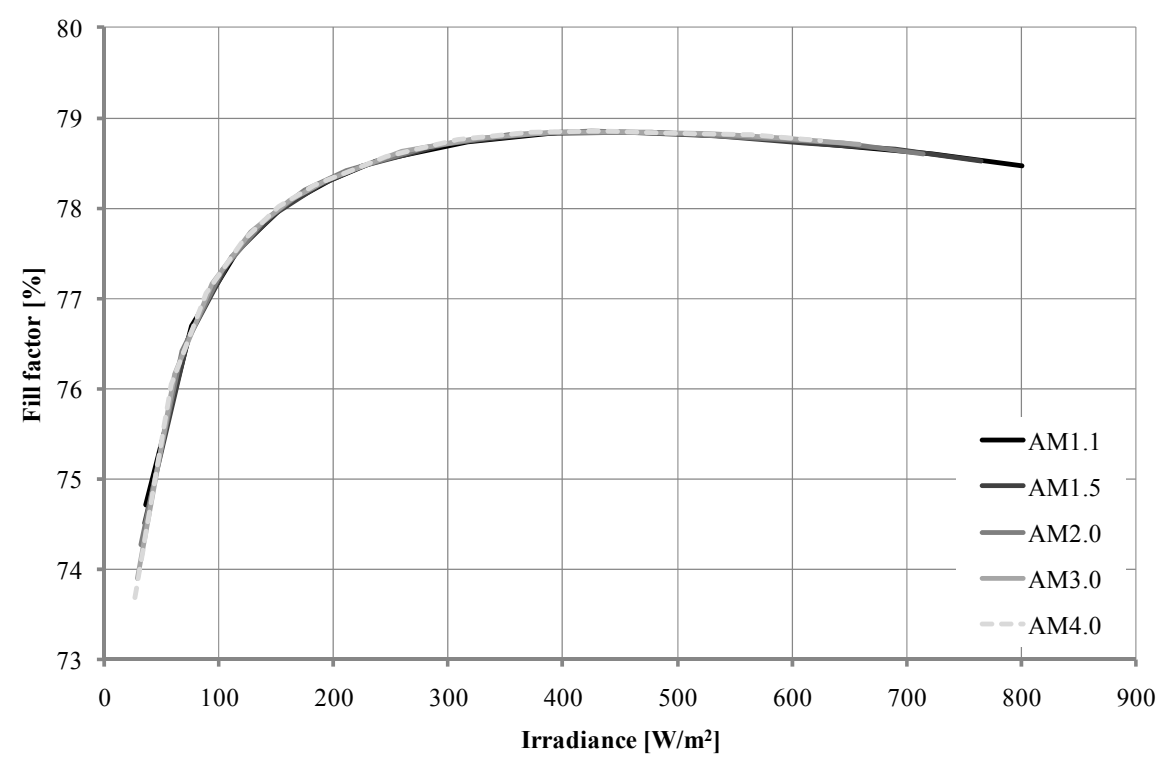

Figure 6: Fill factor versus $\mathrm{G}$ at varying $\mathrm{E}$ at $25^{\circ} \mathrm{C}$; no changes due to spectrum have been observed

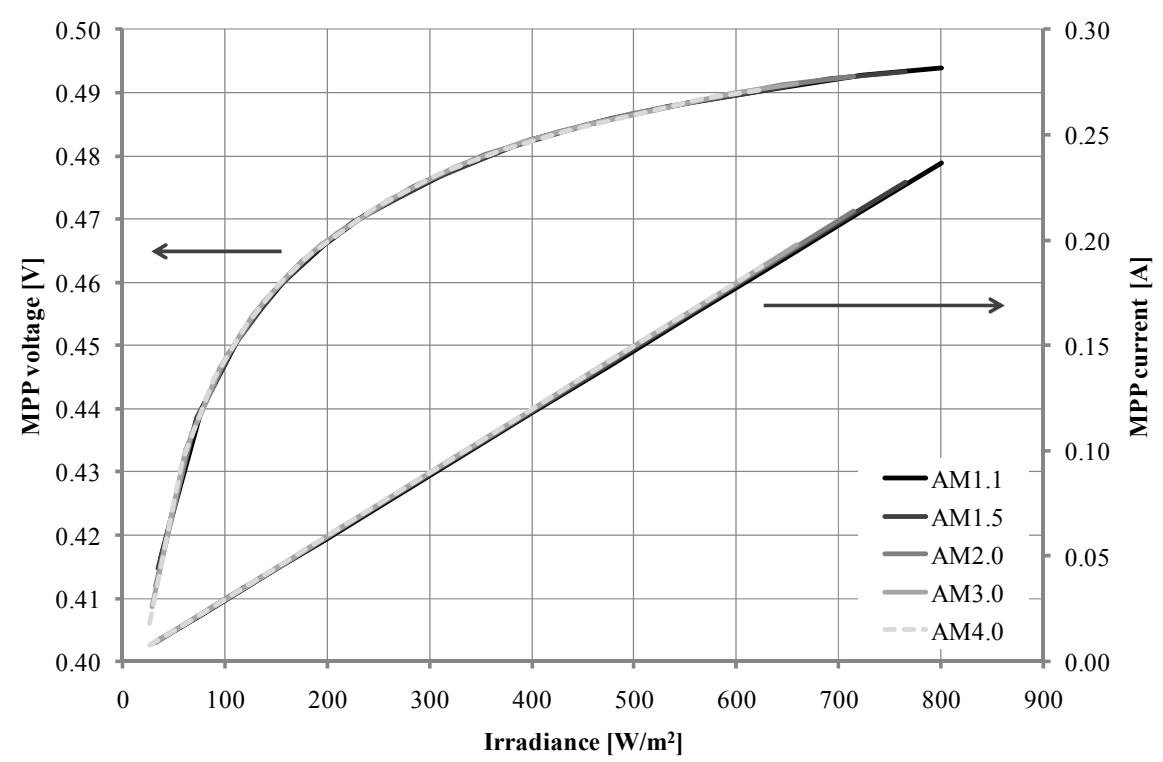

Figure 7: MPP voltage and current behaviour versus irradiance at different sunlight spectra; the slope at MPP current is slightly changing with air mass which is due to the changes in $\mathrm{I}_{\mathrm{SC}}$. $\mathrm{V}_{\mathrm{MPP}}$ is not visibly affected by spectrum

\subsection{Influence of irradiance and temperature}

10 In Figure 8 the influences of temperature and light intensity on the test device's efficiency are shown.

11 As reported in the literature, e.g. [26] or [27], efficiency increases sharply with irradiance in the lower 12 intensity region and flattens out at higher intensities. Efficiency decreases monotonously with 13 temperature as one would expect from basic semiconductor physics. 


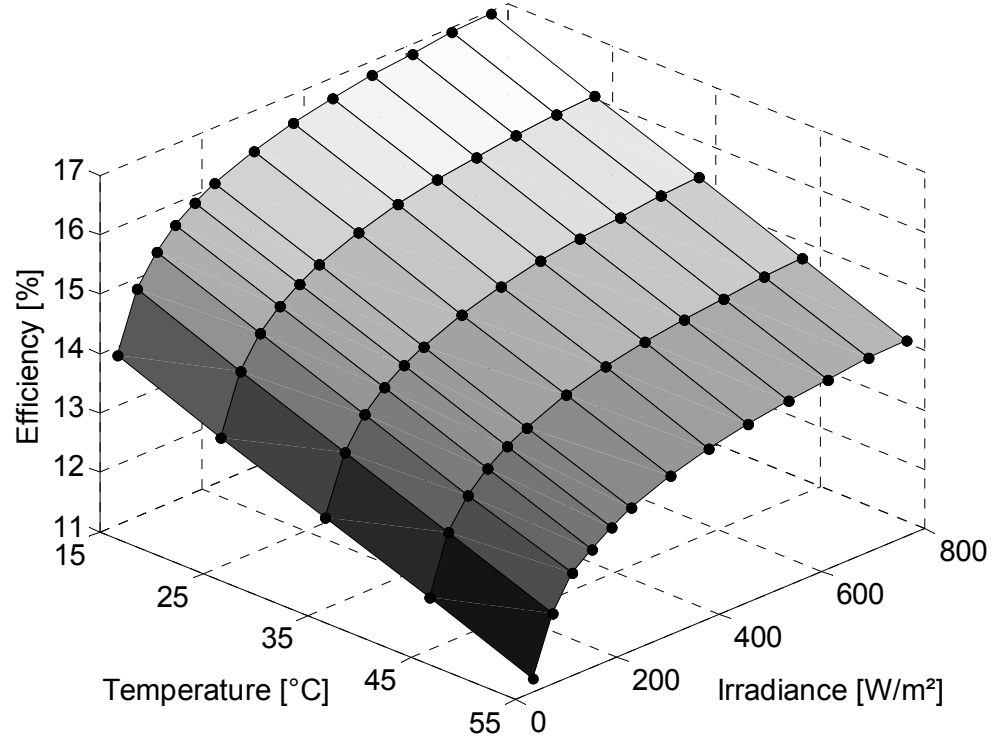

Figure 8: Efficiency versus irradiance and temperature plot at AM1.5 spectrum; dots indicate the actual measurement points

This pattern is largely followed by fill factor. The difference here is that the fill factor reaches a maximum point at $450 \mathrm{~W} / \mathrm{m}^{2}$ and slightly decreases at higher intensities (see Figure 6), indicating that the resistive losses in this device are becoming significant.

The temperature coefficients of the I-V parameters, illustrated in Figure 9, are within expected ranges of a c-Si solar cell and as seen on the CREST outdoor monitoring system data. A small non-linearity is visible for all parameters at low light levels. With increasing intensity the change of the temperature coefficient is getting smaller and becomes linear. The temperature coefficient of power changes by about $20 \%$ in dependence of the intensity, which may be significant in the context of the generally assumed global temperature coefficient for energy modelling. 


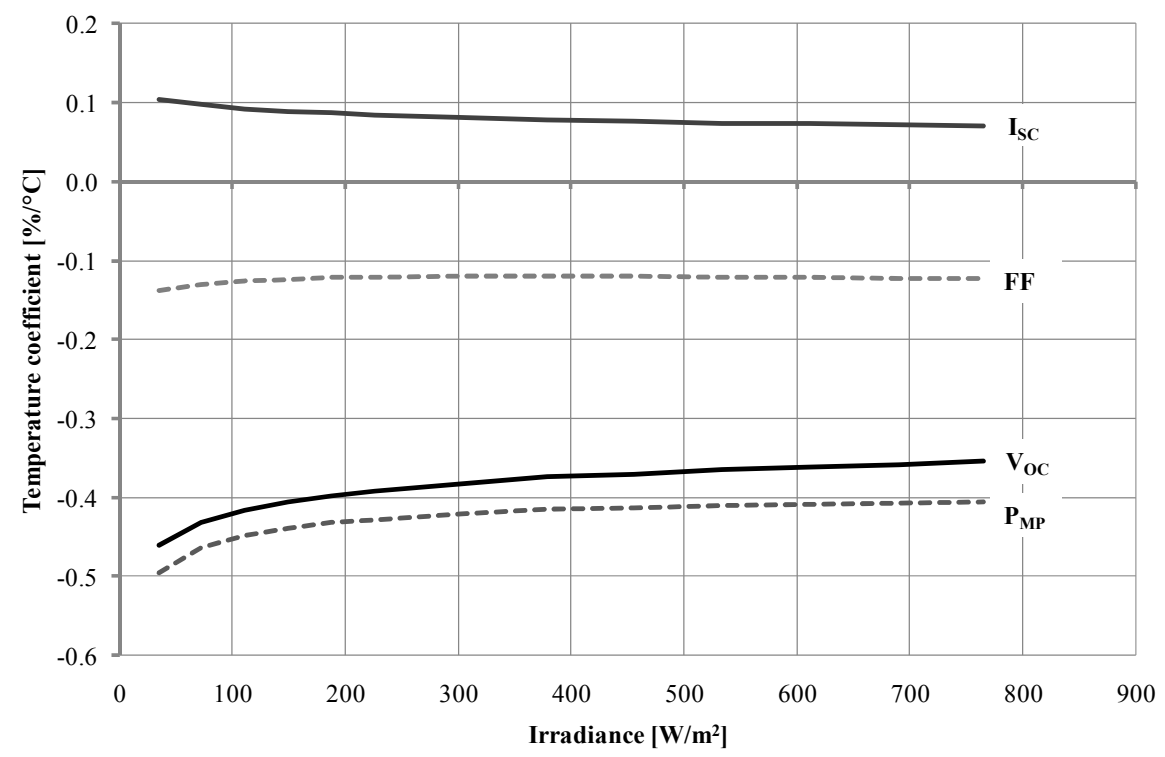

Figure 9: Temperature coefficients of IV parameters extracted under AM1.5 spectrum

\section{Measurement uncertainty analysis}

To determine the robustness of the measurement system, an uncertainty calculation was carried out according to ISO/IEC Guide 98-3 [28], using the approach given in [29]. The influencing factors can be grouped into 4 main sections:

- I-V curve data acquisition and calibration

- Device temperature measurement and conditioning

- Irradiance and spectrum measurement and control

- Device mounting and connections

Uncertainty calculations were made with respect to the I-V curve measurement of the c-Si device at AM1.5 spectrum with highest and lowest intensity. Table 2 summarises the identified uncertainties.

The uncertainties in I-V curve data acquisition (DAQ) are mainly influenced by the absolute measurement card accuracy itself $\left(0.13 \% \mathrm{k}=1\right.$ in $\mathrm{P}_{\mathrm{MAX}}$ at high irradiance) and the calibration of the shunt resistor and amplifiers $\left(0.11 \% \mathrm{k}=1\right.$ in $\mathrm{P}_{\mathrm{MAX}}$ at high irradiance). The contribution of measurement noise and the variations due to changes in operating temperature of the shunt resistor $\left(5^{\circ} \mathrm{C}\right.$ used) are minor.

The second group incorporates uncertainties due to the measurement and control of the device temperature. The use of a K-type thermocouple that was not calibrated $\left( \pm 1.5^{\circ} \mathrm{C}\right)$ introduced the largest uncertainty with $0.35 \%(\mathrm{k}=1)$ on $\mathrm{P}_{\mathrm{MAX}}$ at high irradiance. The non-uniformity over the test device was estimated using a thermal imager at $\pm 0.1^{\circ} \mathrm{C}$ and the temperature increases during measurements due to light induction was set to $0.3^{\circ} \mathrm{C}$ (maximum measured $+0.2^{\circ} \mathrm{C}$ ). 


\section{Indoor Measurement of GTE-matrix for Energy Rating}

1 Uncertainties in irradiance and spectrum are the largest contributors. They affect $\mathrm{I}_{\mathrm{SC}}$ and $\mathrm{P}_{\mathrm{MAX}}$ of the 2 test device. A significant contribution comes from the calibration of the test device for self 3 referencing in the Pasan solar simulator. For this the main influencing factors have been included: reference cell uncertainty of $2.3 \%(\mathrm{k}=2), 0.5 \%$ non-uniformity of light (rectangular distribution) and an estimated $1 \%(\mathrm{k}=2)$ in spectral mismatch. The spatial non-uniformity over the illumination area of the LED-based solar simulator was measured at $1.5 \%$ over $60 \times 60 \mathrm{~mm}^{2}$, this value was used in the uncertainty calculation here. The temporal stability of the light intensity during all measurements was below $0.1 \%$ and has been included in the calculations. Since the test device was used as a self reference and for setting the spectrum in the solar simulator, there is not a direct uncertainty due to spectral mismatch. Nevertheless uncertainties similar to spectral matching arise due to the relative uncertainty in the spectral response (estimated 5\%) and the relative uncertainty in measurements of the simulator light source spectra (estimated 10\%). It has been shown in [30] that the contribution of these factors to the spectral mismatch (or here the difference between the actual $\mathrm{I}_{\mathrm{SC}}$ and the $\mathrm{I}_{\mathrm{SC}}$ that should be measured when the calibrations without uncertainty) is 10 times less. Thus, the uncertainty arising due to the setting of the spectrum is $1.1 \%(\mathrm{k}=1)$. Since self referencing was used only during measurements at $25^{\circ} \mathrm{C}$ to not eliminate temperature effects in the G-T-E matrix measurement an irradiance control repeatability uncertainty of $0.5 \%$ was included (about double of what has been measured in [13]). Device linearity has also been included without reduction factor into the uncertainty calculations, which was measured at $0.4 \%$ at high intensity and $1.7 \%$ at low intensity.

The last uncertainty in the group of irradiance and spectrum that needs to be accounted for is the relative change of spectrum due to the halogen light sources when changing the intensity. As previously mentioned the $G$ vs. $I_{D}$ control of the light sources for calculating the new measurement irradiance was measured with a c-Si reference cell. This meant that the induced current on the test cell from the halogen light sources was in relative terms correct even so the spectrum of the halogen lights shifted to the infrared with lower measurement intensity. Thus, the balance in the current generation of each the light source on the test device was relatively accurate. Self reference furthermore eliminates the uncertainty due to spectral mismatch. A full analysis of the remaining uncertainty due to relative spectral changes has not been done yet for the LED-based solar simulator. It is known that this uncertainty increases with reduction in light intensity, as the light sources are calibrated at maximum intensity. An estimated remaining uncertainty due to spectrum of $2 \%(\mathrm{k}=1)$ on irradiance and short circuit current at $35 \mathrm{~W} / \mathrm{m}^{2}$ measurement irradiance has been included here.

The main uncertainty contribution in the group of device mounting and connections is from the angular distribution of the light. The angle of incoming light is at about $\pm 17^{\circ}$ in the solar simulator because a very large area of light sources with respect to the test device. Assuming a Gaussian distribution and a cosine response this has an impact of $0.67 \%$ uncertainty $(\mathrm{k}=1)$ on irradiance and 
1 current respectively. The influence of cell alignment (estimated at $\pm 1^{\circ}$ ) is negligible in comparison.

2 The uncertainty in the fill factor due to the 4-wire connection of the test device was estimated to be 3 the same as in [29] $(0.45 \%)$.

Table 2: Absolute uncertainty in I-V curve measurements of the G-TE performance matrix at high $\left(765 \mathrm{~W} / \mathrm{m}^{2}\right)$ and low $\left(35 \mathrm{~W} / \mathrm{m}^{2}\right)$ light intensity at AM1.5 spectral setting

\begin{tabular}{|c|c|c|c|c|c|}
\hline \multirow{2}{*}{$\begin{array}{c}\text { Influence \& } \\
\text { intensity }\end{array}$} & \multicolumn{5}{|c|}{ Standard Uncertainty in parameter $(\mathrm{k}=1)[\%]$} \\
\hline & Irradiance & Voltage & Current & Fill Factor & $\mathrm{P}_{\mathrm{MAX}}$ \\
\hline \multicolumn{6}{|c|}{ I-V curve data acquisition and calibration: } \\
\hline high & \pm 0.10 & \pm 0.09 & \pm 0.14 & \pm 0.02 & \pm 0.17 \\
\hline low & \pm 0.94 & \pm 0.12 & \pm 2.03 & \pm 0.08 & \pm 2.03 \\
\hline \multicolumn{6}{|c|}{ Device temperature measurement and conditioning: } \\
\hline high & \pm 0.00 & \pm 0.31 & \pm 0.09 & \pm 0.11 & \pm 0.36 \\
\hline low & \pm 0.00 & \pm 0.41 & \pm 0.13 & \pm 0.12 & \pm 0.44 \\
\hline \multicolumn{6}{|c|}{ Irradiance and spectrum measurement and control: } \\
\hline high & \pm 1.63 & \pm 0.07 & \pm 1.98 & \pm 0.00 & \pm 1.98 \\
\hline low & \pm 2.59 & \pm 0.14 & \pm 2.78 & \pm 0.00 & \pm 2.78 \\
\hline \multicolumn{6}{|c|}{ Device mounting alignment and connections: } \\
\hline high & \pm 0.67 & \pm 0.03 & \pm 0.67 & \pm 0.45 & \pm 0.80 \\
\hline low & \pm 0.67 & \pm 0.04 & \pm 0.67 & \pm 0.45 & \pm 0.80 \\
\hline \multicolumn{6}{|c|}{ Combined uncertainty at high intensity $\left(765 \mathrm{~W} / \mathrm{m}^{2}\right)$ : } \\
\hline $\mathrm{k}=1$ & \pm 1.76 & \pm 0.33 & \pm 2.09 & \pm 0.46 & \pm 2.17 \\
\hline $\mathrm{k}=2$ & \pm 3.52 & \pm 0.67 & \pm 4.18 & \pm 0.93 & \pm 4.34 \\
\hline \multicolumn{6}{|c|}{ Combined uncertainty at low intensity $\left(35 \mathrm{~W} / \mathrm{m}^{2}\right)$ : } \\
\hline $\mathrm{k}=1$ & \pm 2.84 & \pm 0.45 & \pm 3.51 & \pm 0.47 & \pm 3.57 \\
\hline $\mathrm{k}=2$ & \pm 5.67 & \pm 0.90 & \pm 7.01 & \pm 0.94 & \pm 7.13 \\
\hline
\end{tabular}

\section{$\begin{array}{lll}7 & 7 & \text { Discussions }\end{array}$}

8 G-T-E performance matrix measurement results of the c-Si solar cell show a good agreement to 9 reported behaviour of PV modules of the same material outdoors. Which is a good indication that the 10 measurement method presented here is working well and allows indoor based measurement of 11 spectral effects of pertinence to outdoor operation. This is confirmed by the uncertainty analysis. It is 12 shown that low light measurements require further improvements. This is in agreement with recent 13 round robin intercomparisons of test laboratories where the agreement between the participants was 14 significantly worse [31]. The problem is somewhat more convoluted if the spectrum is also changing 15 (deliberately) and further work needs to be done in this area.

16 Using the test device as a self reference for irradiance determination and solar simulator spectrum 17 adjustment did reduce uncertainties in the I-V measurement as the simulator prototype at the time did 18 not allow the positioning of a reference cell next to the test device without introducing errors due to 19 increased light non-uniformity. The self reference method additionally largely eliminates uncertainty 


\section{Indoor Measurement of GTE-matrix for Energy Rating}

1 influences arising due to spectral shift of the halogen lights. Measurements can be improved upon

2 using direct spectral output measurement feedback with a spectroradiometer, which is an entire 3 project in itself and is currently not possible here. This feedback will be needed for measuring multi-

4 junction solar cells as a change in the spectrum of halogen lights changes the junction current balance

5 with changing intensity.

6 From the uncertainty analysis it is apparent that the largest influence on the combined voltage 7 uncertainty during I-V measurements is due to the currently not calibrated temperature sensor used for 8 measurement and device temperature conditioning $\left( \pm 1.5^{\circ} \mathrm{C}\right)$. This is a relatively easy option for 9 improvement, with the possibility of halving the expanded uncertainty in voltage measurements from $10 \pm 0.67 \%(\mathrm{k}=2)$ down to $\pm 0.34 \%$ in the high irradiance case when measuring to an accuracy of $\pm 0.5^{\circ} \mathrm{C}$. 11 Measurement uncertainties in data acquisition make no significant contribution in high irradiance 12 situations. However, this changes when measuring at low intensity as the DAQ card's absolute 13 measurement accuracy at the measurement input range becomes the largest contribution with over $2 \%$ $14(\mathrm{k}=1)$ in the current. An appropriately automated measurement range setting, signal amplification and signal-strength dependent calibration can significantly reduce those additional uncertainties but has yet not been implemented into the system.

The absolute uncertainties in the measurements are comparable to that of major test houses in high irradiance conditions [32]. This changes for low irradiance measurements. Also, with regards to prediction of the energy yield, uncertainties in low irradiance I-V measurements have a less of an impact on the final energy yield, as the majority of the energy is generated in high irradiance conditions (dependent on location).

\section{Conclusions}

A method for a complete indoor characterisation of devices has been presented. A first G-T-E performance matrix measured indoors using an LED-based solar simulator is presented for a c-Si solar cell. It clearly demonstrates that the demonstrated measurement apparatus is capable of measuring the device parameters required for an indoor based approach which includes spectral variations. This opens a new dimension for laboratory based PV device characterisation as it is the first time that sunlight spectra with their variability can be reproduced, meaning that spectral effects on devices can be measured in a controlled environment. The concept is a very promising start for a more accurate energy rating and energy yield prediction especially of thin-film amorphous and multijunction devices, where spectral effects are known to be an issue.

An uncertainty analysis shows that measurements are robust. Uncertainty is comparable to that of commercial test houses in high irradiance conditions. However, it also shows that some improvement and optimisation is needed and possible to provide more accurate data for energy yield prediction, 


\section{Indoor Measurement of GTE-matrix for Energy Rating}

1 especially at low light conditions. Uncertainty contributions have been indentified and will in future

2 be reduced with better calibration accuracies and equipment. In the current measurement set-up with

3 test device self referencing multi-junction devices cannot be measured accurately. Nevertheless, the system provides all functions necessary to measure those more complex devices and initial steps have been taken to make this possible. A new version of the solar simulator for midsize modules is currently under development. This version is planned to use LEDs only, further reducing measurement uncertainties and increasing measurement speed.

\section{Acknowledgements}

This work is supported by Engineering and Physical Sciences Research Council (EPSRC) under grant number EP/D078431/1.

\section{References}

[1] IEC 61215:2005 Crystalline silicon terrestrial photovoltaic (PV) modules — Design qualification and type approval.

[2] G. Friesen, S. Dittmann, S. Williams, R. Gottschalg, H. G. Beyer, A. Guérin de Montgareuil, N. J. C. M. Van Der Borg, A. R. Burgers, R. P. Kenny, T. Huld, B. Müller, C. Reise, J. Kurnik and M. Topic, "Intercomparison of different energy prediction methods within the european project "performance" - results on the 2nd round robin," in Proceedings of the 24th European Photovoltaic Solar Energy Conference, Hamburg, Germany, 2009, pp. 3189 - 3197.

[3] R. Gottschalg, D. G. Infield and M. J. Kearney, "Experimental study of variations of the solar spectrum of relevance to thin film solar cells," Solar Energy Mater. Solar Cells, vol. 79, pp. 527-537, 9/30, 2003.

[4] T. Minemoto, M. Toda, S. Nagae, M. Gotoh, A. Nakajima, K. Yamamoto, H. Takakura and Y. Hamakawa, "Effect of spectral irradiance distribution on the outdoor performance of amorphous Si//thin-film crystalline Si stacked photovoltaic modules," Solar Energy Mater. Solar Cells, vol. 91, pp. 120-122, 1/23, 2007.

[5] R. Gottschalg, T. R. Betts, D. G. Infield and M. J. Kearney, "The effect of spectral variations on the performance parameters of single and double junction amorphous silicon solar cells," Solar Energy Mater. Solar Cells, vol. 85, pp. 415-428, 1/31, 2005.

[6] S. Nann and K. Emery, "Spectral effects on PV-device rating," Solar Energy Mater. Solar Cells, vol. 27, pp. 189-216, 8, 1992.

[7] R. Rüther, G. Kleiss and K. Reiche, "Spectral effects on amorphous silicon solar module fill factors," Solar Energy Mater. Solar Cells, vol. 71, pp. 375-385, 2/15, 2002. 
[8] IEC 60904-7 2008 Photovoltaic devices - Part 7: Computation of the spectral mismatch correction for measurements of photovoltaic devices.

[9] Y. Hishikawa, "Characterization of the Silicon-Based Thin Film Multi-Junction Solar Cells," Proceedings Volume 862 Amorphous and Nanocrystalline Silicon Science and Technology2005, vol. 862, pp. 579-590, 2005.

[10] R. Adelhelm, "Performance and parameter analysis of tandem solar cells using measurements at multiple spectral conditions," Solar Energy Mater. Solar Cells, vol. 50, pp. 185-195, 1998.

[11] A. Nakajima, M. Goto, H. Takata, M. Yoshimi, K. Yamamoto, T. Suezaki, Y. Tawada, T. Meguro, M. Ichikawa, S. Fukuda, K. Hayashi and T. Sawada, "Improvement on actual output power of thin film silicon hybrid module," in Proceedings of the 3rd World Conference on Photovoltaic Energy Conversion, Osaka, Japan, 2003, pp. 1915-1918.

[12] M. Bliss, T. R. Betts and R. Gottschalg, "An LED-based photovoltaic measurement system with variable spectrum and flash speed," Solar Energy Mater. Solar Cells, vol. 93, pp. 825-830, 6, 2009.

[13] M. Bliss, S. Wendlandt, T. R. Betts and R. Gottschalg, "Towards a higher power, all LED solar simulator closely matching realistic solar spectra," in Proceedings of the 24th European

[14] IEC 60904-9 2007 photovoltaic devices - part 9: Solar simulator performance requirements.

[15] C. A. Gueymard, "Parameterized transmittance model for direct beam and circumsolar spectral irradiance," Solar Energy, vol. 71, pp. 325-346, 11, 2001.

[16] IEC 61853 photovoltaic (PV) module performance testing and energy rating (draft standard).

[17] K. Heidler and B. Muller-Bierl, "Measurement of multi-junction solar cells," in Proceedings of the 10th European Photovoltaic Solar Energy Conference, Lisbon, Portugal, 1991, pp. 111 114.

[18] K. Heidler, A. Schonecker, B. Muller-Bierl and K. Bucher, "Progress in the measurement of multi-junction devices at ISE [solar cell testing]," in Photovoltaic Specialists Conference, 1991., Conference Record of the Twenty Second IEEE, 1991, pp. 430-435 vol.1.

[19] M. Meusel, R. Adelhelm, F. Dimroth, A. W. Bett and W. Warta, "Spectral Mismatch Correction and Spectrometric Characterization of Monolithic III-V Multi-junction Solar Cells," PROGRESS IN PHOTOVOLTAICS, vol. 10; pp. 243-256, 2002.

[20] IEC 60904-3 2008 Photovoltaic devices - Part 3: Measurement Principles for Terrestrial PV Solar Devices with Reference Spectral Irradiance Data.

[21] C. J. Hibberd, M. Bliss, H. M. Upadhyaya and R. Gottschalg, "Characterisation of a filter-based external quantum efficiency measurement system," in Proceedings of the 5th Photovoltaic Science Application and Technology Conference (PVSAT-5), Wrexham, UK, 2009, pp. 239 242.

[22] IEC 60904-10 2009 Photovoltaic devices - Part 10: Methods of linearity measurement. Photovoltaic Energy Conversion, Conference Record of the 2006 IEEE 4th World Conference on, 2006, pp. 2177-2180. 
[24] R. P. Kenny, A. Ioannides, H. Müllejans, W. Zaaiman and E. D. Dunlop, "Performance of thin film PV modules," Thin Solid Films, vol. 511-512, pp. 663-672, 7/26, 2006.

[25] D. L. King, J. A. Kratochvil and W. E. Boyson, "Stabilization and performance characteristics of commercial amorphous-silicon PV modules," in Photovoltaic Specialists Conference, 2000. Conference Record of the Twenty-Eighth IEEE, 2000, pp. 1446-1449.

[26] J. A. Eikelboom and M. J. Jansen. (2000, Characterisation of PV modules of new generations results of tests and simulations. The Netherlands Energy Research Foundation.

[27] O. Midtgard, T. O. Sætre, G. Yordanov, A. G. Imenes and C. L. Nge, "A qualitative examination of performance and energy yield of photovoltaic modules in southern Norway," Renewable Energy, vol. 35, pp. 1266-1274, 6, 2010.

[28] ISO/IEC guide 98-3:2008 uncertainty of measurement - part 3: Guide to the expression of uncertainty in measurement (GUM:1995).

[29] H. Mullejans, W. Zaaiman and R. Galleano, "Analysis and mitigation of measurement uncertainties in the traceability chain for the calibration of photovoltaic devices," MEASUREMENT SCIENCE AND TECHNOLOGY, vol. 20, 2009.

[30] H. Field and K. Emery, "An uncertainty analysis of the spectral correction factor," in Photovoltaic Specialists Conference, 1993., Conference Record of the Twenty Third IEEE, 1993, pp. 1180-1187.

[31] W. Herrmann, "Leistungscharacterisierung und vergleichbarkeit von messergebnissen," in 6. Workshop Photovoltaische Modultechnik, 2009.

[32] R. Haselhuhn, "Modultests aus anwendersicht," in 6. Workshop Photovoltaische Modultechnik, 2009.

\footnotetext{
${ }^{i}$ European Solar Test Installation of the Joint Research Centre of the European Commission, which is one of the few primary calibration laboratories world wide
} 